University of New Hampshire

University of New Hampshire Scholars' Repository

$2-8-2004$

\title{
High resolution measurements of kinetic energy release distributions of neon, argon, and krypton cluster ions using a three sector field mass spectrometer
}

K. Gluch

S. Matt-Leubner

L. Michalak

Olof E. Echt

University of New Hampshire, Olof.Echt@unh.edu

A. Stamatovic

See next page for additional authors

Follow this and additional works at: https://scholars.unh.edu/physics_facpub

Part of the Physics Commons

\section{Recommended Citation}

The following article appeared in J. Chem. Phys. 120, 2686 (2004); doi: 10.1063/1.1636459 and may be found at http://dx.doi.org/10.1063/1.1636459.

This Article is brought to you for free and open access by the Physics at University of New Hampshire Scholars' Repository. It has been accepted for inclusion in Physics Scholarship by an authorized administrator of University of New Hampshire Scholars' Repository. For more information, please contact Scholarly.Communication@unh.edu. 


\section{Authors}

K. Gluch, S. Matt-Leubner, L. Michalak, Olof E. Echt, A. Stamatovic, P. Scheier, and T. D. Mark 


\section{AIP $\begin{gathered}\text { molowanat } \\ \text { chemical Physics }\end{gathered}$}

High resolution measurements of kinetic energy release distributions of neon, argon, and krypton cluster ions using a three sector field mass spectrometer

K. Głuch, S. Matt-Leubner, L. Michalak, O. Echt, A. Stamatovic et al.

Citation: J. Chem. Phys. 120, 2686 (2004); doi: 10.1063/1.1636459

View online: http://dx.doi.org/10.1063/1.1636459

View Table of Contents: http://jcp.aip.org/resource/1/JCPSA6/v120/i6

Published by the American Institute of Physics.

Additional information on J. Chem. Phys.

Journal Homepage: http://jcp.aip.org/

Journal Information: http://jcp.aip.org/about/about_the_journal

Top downloads: http://jcp.aip.org/features/most_downloaded

Information for Authors: http://jcp.aip.org/authors

\section{ADVERTISEMENT}
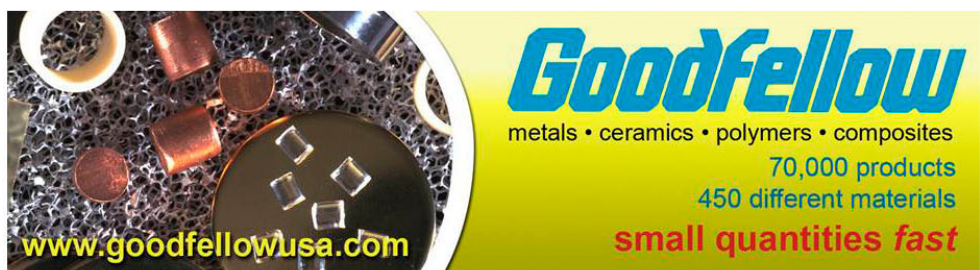


\title{
High resolution measurements of kinetic energy release distributions of neon, argon, and krypton cluster ions using a three sector field mass spectrometer
}

\author{
K. Głuch, ${ }^{\text {a) }}$ S. Matt-Leubner, ${ }^{\text {b) }}$ L. Michalak, ${ }^{\text {a) }}$ O. Echt, ${ }^{\text {c) }}$ A. Stamatovic, ${ }^{\text {d) }}$ P. Scheier, \\ and T. D. Märk ${ }^{\mathrm{e}}$ \\ Institut für Ionenphysik, Leopold-Franzens Universität, Innsbruck, Technikerstr. 25, \\ A-6020 Innsbruck, Austria
}

(Received 25 September 2003; accepted 3 November 2003)

\begin{abstract}
Using a newly constructed three sector field mass spectrometer (resulting in a BE1E2 field configuration) we have measured the kinetic energy release distributions of neon, argon, and krypton cluster ions. In the present study we used the first two sectors, B and E1, constituting a high resolution mass spectrometer, to select the parent ions in terms of mass, charge, and energy, and studied the decay of those ions in the third field free region. Due to the improved mass resolution we were able to extend earlier studies carried out with a two sector field machine, where an upper size limit arose from the fact that several isotopomers contribute to a decaying parent ion beam when the cluster size exceeds a certain value. Furthermore we developed a new data analysis. It allows us to model also fragment ion peaks that are a superposition of different decay reactions and thus we can determine the average kinetic energy release for all decay reactions of a given cluster ion. In a further step we used these results to determine the binding energies of cluster ions $\operatorname{Rg}_{n}(n \geqslant 10)$ by applying finite heat bath theory. The smaller sizes have not been included in this analysis, because the validity of finite heat bath theory becomes questionable below $n \approx 10$. The present average kinetic energy releases and binding energies are compared with other experiments and various calculations. (C) 2004 American Institute of Physics. [DOI: 10.1063/1.1636459]
\end{abstract}

\section{INTRODUCTION}

If cluster ions decay on the $\mu$ s time scale, the dominant mechanism is very likely vibrational predissociation. ${ }^{1}$ It is then possible to model the fragmentation process by statistical theories like, for example, Rice-Ramsperger-KasselMarcus (RRKM) theory, ${ }^{2,3}$ sometimes also called quasiequilibrium theory (QET). One can calculate, for example, the kinetic energy release as a function of time and compare the results with measurements in order to prove the statistical nature of the fragmentation process. This is important because it is not clear from the beginning, whether vibrational predissocation is the major decay mechanism at all times and for all systems. It is also possible that excited cluster ions relax by emitting electrons or photons. In particular, in the $\mu$ s time regime radiative cooling may be as important as the dissociative cooling, for example in the case of the fullerenes. ${ }^{4,5}$ One of the first studies dealing with the statistical description of cluster ion fragmentation was carried out by Engelking. ${ }^{6}$ Engelking analyzed the evaporative lifetime

\footnotetext{
a) Permanent address: Institute of Mathematics, Physics and Informatics, Maria Curie-Sklodowka University, Lublin 20-031, Poland.

b) Author to whom correspondence should be addressed. Electronic mail: sara.matt@uibk.ac.at

${ }^{c}$ Permanent address: Physics Department, University of New Hampshire, Durham, New Hampshire 03824.

d)Permanent address: Faculty of Physics, P.O. Box 638, Yo-11001 Beograd, Yugoslavia.

e) Also adjunct Professor at Department of Plasma Physics, Comenius University, SK-84248 Bratislava, Slovak Republic.
}

and the average kinetic energy release measurements carried out by Stace et al. ${ }^{7,8}$ for argon and $\mathrm{CO}_{2}$ cluster ions in order to determine the binding energy of the decaying clusters using a RRK (Ref. 2) type model. The same method was used by the group of Castleman to determine binding energies of ammonia cluster ions. ${ }^{9}$ In other work, Castleman et al. used the so-called finite heat bath theory developed by Klots $^{10}$ to analyze their data on ammonia and xenon cluster ions ${ }^{11,12}$ and to derive the corresponding binding energies. In finite heat bath theory the relative binding energies of a series of decaying cluster ions can be determined by measuring the metastable fractions and calibrating them using estimated heat capacities and frequency factors. In order to obtain absolute values for the binding energies in the frame of finite heat bath theory it is necessary to determine experimentally the average kinetic energy releases, because it has been proposed by Lifshitz and Louage ${ }^{13}$ that the average energy, with which a monomer leaves the cluster, is a measure of the temperature of the transition state.

Extending our previous investigations ${ }^{14,15}$ on this subject, in the present paper we measured the kinetic energy release by using the mass analyzed ion kinetic energy (MIKE) scan technique and determined absolute binding energies for neon, argon, and krypton cluster ions $\operatorname{Rg}_{n},(n$ $\geqslant 10$ ) by using finite heat bath theory. The results on $\langle\mathrm{KER}\rangle_{\mathrm{s}}$ of smaller cluster ions $\left(\operatorname{Rg}_{n}\right.$ with $\left.n<10\right)$ have not been treated with FHBT because various experiments, ${ }^{16,17}$ including also the present one, have shown that clusters or molecules with less than about ten constituents do not always 


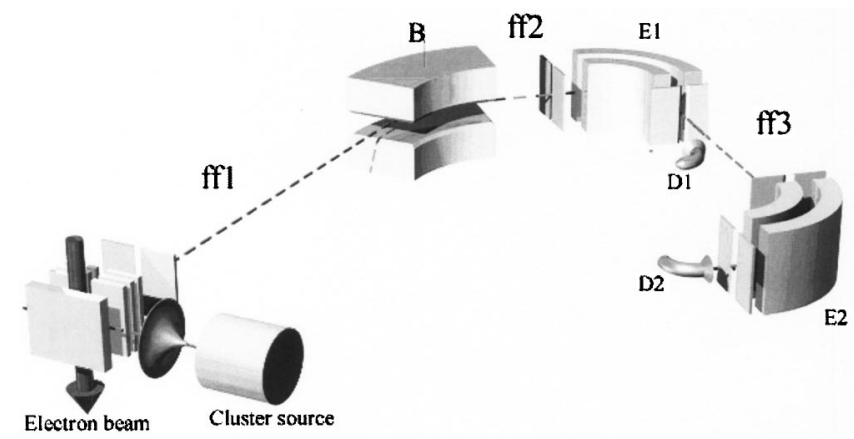

FIG. 1. Schematic drawing of the three sector field mass spectrometer. ff1, $\mathrm{ff} 2$, and $\mathrm{ff} 3$ are the three field free regions, B is the magnetic sector field, E1 and $\mathrm{E} 2$ are the electrostatic analyzers, and D1 and D2 are the two detectors.

behave statistically. In another type of experiment carried out with the present set up we investigated the time dependence of the kinetic energy release of propane $(11 \text { atoms })^{18}$ and it turned out that one can describe this system excellently with finite heat bath theory.

Our previous experiments on neon and krypton cluster ions with a two sector field spectrometer ${ }^{15}$ were limited to relatively small cluster ions, because the "mass" selection by the magnetic field alone does not permit selection of individual isotopomers for elements like $\mathrm{Kr}$ that have many naturally occurring isotopes, except for very small cluster sizes. The present results on binding energies for neon are compared with experimental results from Hiraoka ${ }^{19}$ and recent results from Gianturco and co-workers ${ }^{20}$ using different interaction potentials within the cluster ions, for argon with results from Hiraoka ${ }^{21}$ and calculations of Böhmer and Peyerimhoff $^{22}$ and for krypton with results obtained recently by Kalus et al., ${ }^{23}$ who calculated evaporation energies using variations of the diatomics-in-molecules (DIM) model.

\section{EXPERIMENT}

The apparatus consists of a high-resolution double focusing two sector-field mass spectrometer of reversed NierJohnson-type geometry (see Fig. 1) combined with an additional electrostatic analyzer. Inert gas clusters are produced by expanding neon, argon or krypton from a stagnation chamber with a pressure of about 1 bar through a nozzle (diameter $20 \mu \mathrm{m}$ ) into a vacuum of about $10^{-7}$ Torr. The ensuing neutral clusters are ionized by an electron beam of variable energy and current. The cluster ions produced are then extracted by an electric field and accelerated by $3 \mathrm{kV}$ into the spectrometer. They pass through the first field free region (ff1, length $61 \mathrm{~cm}$ ), are then momentum-analyzed by a magnetic sector field $\mathrm{B}$, enter a second field-free region (ff2, length $33.3 \mathrm{~cm}$ ), pass through a first $90^{\circ}$ electric sector field (E1), are then either detected by a channeltron type electron multiplier (D1) which can be moved into the ion beam, or enter the third field-free region (ff3, length $92 \mathrm{~cm}$ ), pass through a second $90^{\circ}$ sector field (E2) and are finally detected by a channeltron-type electron multiplier (D2). The recent addition of this third sector field (E2) (Ref. 18) enables us to study with a high mass and energy resolution the kinetic energy release distributions (KERD) of various cluster ions by using the so-called mass analyzed ion kinetic energy (MIKE) scan technique. ${ }^{24}$

MIKE spectra are recorded as follows: The magnet is tuned to the mass to charge ratio of the parent ion, mass $m_{p}$ and charge state $z$, and the first electrostatic sector field voltage is set to select ions with kinetic energy corresponding to the accelerating voltage $U_{\text {acc }}$ used in the ion source, while the second electrostatic sector field voltage (ESF voltage) $U$ is scanned. Stable singly charged ions will have a kinetic energy of $3 \mathrm{kV}$ and pass at the nominal second sector field voltage of $U_{p}=511 \mathrm{~V}$. Fragment ions (mass $m_{f}$ ), formed in ff 3 in a spontaneous decay reaction, will then pass at a voltage

$$
U_{f}=\frac{m_{f}}{m_{p}} U_{p} .
$$

This equation relates the position of a fragment ion peak to the position of the parent ion peak in a MIKE spectrum. If no kinetic energy were released in the decay reaction, the fragment ion peak would have the same shape as the parent ion peak, only scaled by the ratio of the electric sector field voltages $U_{f} / U_{p}$. For a unimolecular dissociation reaction with a Gaussian MIKE peak the average kinetic energy can be extracted ${ }^{25}$ from

$$
\langle\mathrm{KER}\rangle=\frac{z_{2}^{2} m_{1}^{2} U_{\mathrm{acc}}}{16 z_{1} m_{2} m_{3}}\left(\frac{\Delta U}{U_{p}}\right)^{2} \times 2.16,
$$

where $\Delta U$ is the full-width at half-maximum (FWHM) of the fragment ion peak corrected for the finite width of the parent ion peak by deconvoluting the former with the latter. $m_{1}, m_{2}$, and $m_{3}$ are the masses of parent ion, first fragment ion, and second fragment, respectively, and $z_{1}$ and $z_{2}$ are the charge states of the parent ion and the charged fragment ion. The factor of 2.16 has to used if one uses for the calculation of the $\langle\mathrm{KER}\rangle$ the FWHM, which is easier to measure, and not the width at $22 \%$ height of the peak as would be correct for a Gaussian fragment ion peak. ${ }^{25}$

Gaussian peaks are observed when the decaying ensemble is prepared in a way that the internal energy is equally partitioned over all degrees of freedom and no reverse activation barrier prevents the production of ions with low kinetic energies. The cluster ions are produced with a distribution of internal energies in the ion source, but only a distinct subensemble of these ions will decay in the experimental time window of about 20-160 $\mu$ s, the length of the time window depending on the mass of the selected cluster ion which we sample by the MIKE scan technique in ff3.

In the present study we use a modified MIKE method, because we selected the parent ions not only with a magnetic field, but also with the electrostatic analyzer E1. This has several advantages in comparison with the standard MIKE technique. $^{24}$

First, in a standard MIKE spectrum, where parent ions $m_{p}$ are selected with the magnetic analyser only in terms of their momentum-not their mass to charge ratio, decay reactions occurring in ff 2 are recorded by E1. Ions $m_{1}$ with $m_{1}$ $>m_{p}$ coming from the ion source and decaying in ff1 will also contribute to the MIKE spectrum if they have the same 
momentum as $m_{p}$ coming from the ion source, i.e., if their apparent mass $m^{*}=m_{2}^{2} / m_{1}$ is approximately equal to $m_{p}$. In contrast, MIKE spectra recorded in $\mathrm{ff} 3$ are free of these contaminations because any fragment ion will have a smaller kinetic energy than the parent ions; they will not pass through the energy selective E1. ${ }^{26}$

Second, we can now also carry out measurements for cluster ions whose monomers consist of many isotopes like, for example, krypton. If one selects with the magnetic field one specific parent ion mass, only this parent ion mass will pass the aperture between the magnetic and electrostatic analyzer and appear in a mass spectrum as clearly resolved. However, neighboring masses will also enter the second field free region and they may decay during their flight through ff2. Some of these decay products can contribute to the MIKE peak. The resulting superposition of different reaction products (from different parent ion masses) makes it nearly impossible to analyze accurately the MIKE peak, especially because an additional difficulty arises from the fact that larger cluster ions have different isotopic compositions even if they have the same mass to charge ratio. We call such ions of the same mass to charge ratio but different composition "isotopomers." Due to different compositions of the selected parent ion the evaporated monomers can have different masses leading to different partial MIKE peaks. This leads to an additional modification of the shape of the MIKE peak, but if all isotopomer probabilities are taken into account in the analysis, it is possible (see below) to calculate the various positions in the MIKE spectrum and the relative abundances of the various contributions and thus disentangle the different contributions.

\section{DATA ANALYSIS}

In all experiments considered here, an analytical expression is required to relate the unimolecular rate constant $k$ either to the vibrational excitation energy $E$ of the parent molecule or to a canonical temperature $T_{b} . k(E)$ is usually calculated from the microcanonical RRKM expression, ${ }^{27}$

$$
k(E)=\frac{\alpha G^{*}\left(E-E_{c}\right)}{h N(E)},
$$

where $\alpha$ is the reaction path degeneracy, $E_{c}$ is the critical energy for the reaction, $G^{*}\left(E-E_{c}\right)$ is the number of states of the activated complex, $N(E)$ is the density of states, and $h$ is Planck's constant.

Alternatively, the rate constant can be obtained from the Arrhenius relation

$$
k\left(T_{b}\right)=A \exp \left(-\frac{E_{a}}{k_{B} T_{b}}\right),
$$

where $k_{B}$ is Boltzmann's constant, and $T_{b}$ is the temperature of a fictitious heat bath for which the rate $k\left(T_{b}\right)$ would equal $k(E) . T_{b}$ is linked to the transition state temperature $T^{\#}$ of the reaction by

$$
T_{b}=T^{\#} \frac{\exp (\gamma / C-1)-1}{\gamma / C-1},
$$

where $C$ is the vibrational heat capacity in units of $k_{B}$ minus 1 of the parent ion $^{28}$ and $\gamma$ is defined by

$$
\gamma=\ln (A)-\ln \left(k\left(T_{b}\right)\right) .
$$

The $A$ factor in Eq. (4) lumps together several poorly known quantities, including the vibrational partition functions of parent and fragment. ${ }^{29}$ Many authors who applied Eq. (4) assumed a value of $A \approx 1.6 \times 10^{15} \mathrm{~s}^{-1}$ which had been shown by $\mathrm{Klots}^{29}$ to apply to a variety of atomic clusters over a wide range of cluster sizes.

For an evaporative ensemble which is characterized by a broad range of excitation energies, the rate constant of the subensemble that contributes to the experimental signal measured at time $t$ after excitation is given by $k=1 / t . t=10 \mu \mathrm{s}$ is often considered a typical experimental time window in which case $A=1.6 \times 10^{15} \mathrm{~s}^{-1}$ is corresponding to $\gamma=23.5$.

However, the relation between the average kinetic energy $\langle\mathrm{KER}\rangle$ and the transition state temperature $T^{\#}$ is not unique. In his model free approach, Klots ${ }^{28}$ suggested that the KERD for the decay of atomic cluster ions may be expressed as

$$
f(\mathrm{KER})=\mathrm{KER}^{\ell} \exp \left(\frac{-\mathrm{KER}}{k_{B} T^{\#}}\right) .
$$

$\ell$ is bound by $0 \leqslant \ell \leqslant 1$, hence $\langle\operatorname{KER}\rangle=(1+\ell) k_{B} T^{\#}$, and $k_{B} T^{\#} \leqslant\langle\mathrm{KER}\rangle \leqslant 2 k_{B} T^{\#}$. In our data analysis $\ell$ was assumed to be 0.5 because in our experiment all recorded MIKE peaks are Gaussian (for more details on this choice, see Ref. 30).

The standard procedure of evaluating data consists of (i) removing statistical noise from the MIKE peak, (ii) deconvoluting the fragment ion peak with the parent ion peak, (iii) differentiating the signal with respect to the sector field voltage, and finally (iv) converting the sector-field voltage scale to the kinetic energy scale. The differentiation requires extreme smoothing of the raw data; from our experience we know that this procedure easily leads to distortions of the resulting KERD's. Therefore, in order to avoid this problem, we have inverted the procedure. The experimental data are not manipulated. Instead, in an iterative fashion, we start with a hypothetical shape for the KERD [in the present case using Eq. (7) with $l=0.5]$. This KERD is integrated, the energy values are transformed to the sector field voltage, and the resulting theoretical MIKE peak is convoluted with the parent ion peak. Then the normalized sum of weighted square deviations between the theoretical and the experimental MIKE peak is computed to evaluate the fit.

For elements rich in isotopes a complication arises from the presence of isotopomers that have identical nominal mass. For example, $\mathrm{Kr}$ contains six naturally occurring isotopes $(78,80,82,83,84$, and 86$)$. In principle, one may mass-select isotopically pure parent cluster ions such as $\mathrm{Kr}_{8}^{+}$ of mass $8 \times 78 \mathrm{Da}$, or $8 \times 86 \mathrm{Da}$, but those isotopomers will have exceedingly low intensity. Therefore, in practice, one will have to select a mass near the centroid of the $\mathrm{Kr}_{8}^{+}$peak. This parent ion will, with various probabilities, contain any of the naturally occurring isotopes. Unimolecular loss of a monomer will then give rise to six distinct metastable peaks in the MIKE spectrum (corresponding to six different iso- 
TABLE I. Relative probabilities of the seventeen different isotopomers of $\mathrm{Kr}_{8}^{+}$at mass $671 \mathrm{Da}$. The sum of all relative probabilities of $\mathrm{Kr}_{8}^{+}$cluster ions with masses between 624 and $688 \mathrm{Da}$ is equal to one. The last two rows show the total relative probabilities that $\mathrm{Kr}_{8}^{+}(671 \mathrm{Da})$ contains a given isotope, and the electric sector field voltage of the metastable peak in the MIKE spectrum corresponding to loss of that isotope.

\begin{tabular}{|c|c|c|c|c|c|c|c|}
\hline \multirow{2}{*}{$\begin{array}{l}\text { Isotopomer } \\
\quad \text { (label) }\end{array}$} & \multirow[b]{2}{*}{ Probability } & \multicolumn{6}{|c|}{ Isotopic composition } \\
\hline & & ${ }^{78} \mathrm{Kr}$ & ${ }^{80} \mathrm{Kr}$ & ${ }^{82} \mathrm{Kr}$ & ${ }^{83} \mathrm{Kr}$ & ${ }^{84} \mathrm{Kr}$ & ${ }^{86} \mathrm{Kr}$ \\
\hline 1 & 0.04666 & 0 & 0 & 1 & 1 & 5 & 1 \\
\hline 2 & 0.01799 & 0 & 0 & 0 & 1 & 7 & 0 \\
\hline 3 & 0.01441 & 0 & 0 & 2 & 1 & 3 & 2 \\
\hline 4 & 0.007777 & 0 & 0 & 0 & 3 & 4 & 1 \\
\hline 5 & 0.006867 & 0 & 1 & 0 & 1 & 4 & 2 \\
\hline 6 & 0.002882 & 0 & 0 & 1 & 3 & 2 & 2 \\
\hline 7 & 0.001697 & 0 & 1 & 1 & 1 & 2 & 3 \\
\hline 8 & $5.93 \mathrm{E}-4$ & 0 & 0 & 3 & 1 & 1 & 3 \\
\hline 9 & $4.32 \mathrm{E}-4$ & 1 & 0 & 0 & 1 & 3 & 3 \\
\hline 10 & $1.13 \mathrm{E}-4$ & 0 & 1 & 0 & 3 & 1 & 3 \\
\hline 11 & $5.93 \mathrm{E}-05$ & 0 & 0 & 2 & 3 & 0 & 3 \\
\hline 12 & $5.76 \mathrm{E}-05$ & 0 & 0 & 0 & 5 & 1 & 2 \\
\hline 13 & $4.00 \mathrm{E}-05$ & 1 & 0 & 1 & 1 & 1 & 4 \\
\hline 14 & $2.62 \mathrm{E}-05$ & 0 & 1 & 2 & 1 & 0 & 4 \\
\hline 15 & $2.50 \mathrm{E}-05$ & 0 & 2 & 0 & 1 & 1 & 4 \\
\hline 16 & $1.3 \mathrm{E}-06$ & 1 & 0 & 0 & 3 & 0 & 4 \\
\hline 17 & 4.7E-07 & 1 & 1 & 0 & 1 & 0 & 5 \\
\hline \multirow{2}{*}{\multicolumn{2}{|c|}{$\begin{array}{l}\text { Total probability, } p_{j} \\
\text { Position in MIKE scan, } x_{j}\end{array}$}} & 0.00047 & 0.009 & 0.082 & 0.122 & 0.472 & 0.112 \\
\hline & & 451.599 & 450.076 & 448.553 & 447.791 & 447.030 & 445.507 \\
\hline
\end{tabular}

topes which can be ejected in the decay) that cannot be fully resolved because of the broadening caused by the KER.

In order to extract the width of these metastable peaks from the MIKE spectra, we compute their position (ESF voltages) and relative probabilities based on mass and natural abundance of isotopes, and mass and ESF voltage of the selected parent ion. For the purpose of illustration, Table I lists all seventeen $\mathrm{Kr}_{8}^{+}$isotopomers of mass $671 \mathrm{Da}$. For each of them we calculate their relative contributions to the parent ion (column 2). From these values we compute the total (relative) probabilities that the $\mathrm{Kr}_{8}^{+}(671 \mathrm{Da})$ parent ion contains a monomer of mass $78 \mathrm{Da}, 80 \mathrm{Da}$, etc. The last two rows list these probabilities $p_{i}$ together with the computed sector field voltages $x_{i}$ for the metastable peaks [Eq. (1)] that arise from the loss of one of those isotopes.

The measured MIKE spectrum of $\mathrm{Kr}_{8}^{+}(671 \mathrm{Da})$ is then analyzed by fitting a set of six Gaussians of known position and relative amplitude and a common width $w$ (standard deviation),

$$
G(x)=a\left[\sum_{i} p_{i} e^{-\left[(x-x i)^{2} / 2 w^{2}\right]}\right],
$$

where $x$ is the sector field voltage. The sum is over all isotopes contained in the mass-selected parent ion, i.e., six in the example discussed above. The expression involves only two fitting parameters, amplitude $a$ and width $w$. From $w$ we derive the average KER $(\langle\mathrm{KER}\rangle)$ as explained in our discussion of Eq. (2).

For large clusters we do not have unit mass resolution in the parent ion spectrum, because we cannot close the slits that define the mass resolution completely without losing the ion signal, and the expression in Eq. (8) has to be summed over contributions from adjacent parent masses (670 and 672 Da in the example), each with their own set of $p_{j}$ and $x_{j}$ values calculated as described above.

\section{RESULTS AND DISCUSSION}

We were not able in our earlier studies ${ }^{15}$ to perform KERD and binding energy measurements for rare gas cluster ions above a certain size due to interferences from decays in the first field free region, and the presence of isotopomers. In contrast, the present three sector field set-up allows us to block any fragment ions that are formed in the first field free region and, due to the improved mass resolution, to extend the analysis to larger clusters. Furthermore, we quantitatively model the contribution of isotopomers of identical mass but different isotopic composition.

In Fig. 2 we show, as an example, a mass spectrum for krypton cluster ions obtained with the three sector field mass spectrometer: (a) a complete $\mathrm{Kr}_{n}^{+}$mass spectrum up to $n$ $\leqslant 20$, (b) a close up of a high resolution mass spectrum of $\mathrm{Kr}_{3}^{+}$where the resolving power is high enough to resolve isotopomers of $\mathrm{Kr}_{3}^{+}$that have different nominal mass, and (c) a spectrum of $\mathrm{Kr}_{8}^{+}$where isotopomers of different nominal mass are seen to overlap. (The low intensity of large cluster ions makes it impossible to record these spectra with very narrow slits.)

In Fig. 3 we show a representative MIKE scan for $\mathrm{Kr}_{8}^{+}$ cluster ions. The mass of $\mathrm{Kr}_{8}^{+}$spans a range from 624 to 688 $\mathrm{Da}$, depending on the isotopic composition. We selected the parent ion with mass $671 \mathrm{Da}$; it appears at a sector field voltage of $511 \mathrm{~V}$ while its various metastable fragment ions appear at voltages ranging from 444 to $452 \mathrm{~V}$. As expected from the computed probabilities for the various isotopomers 

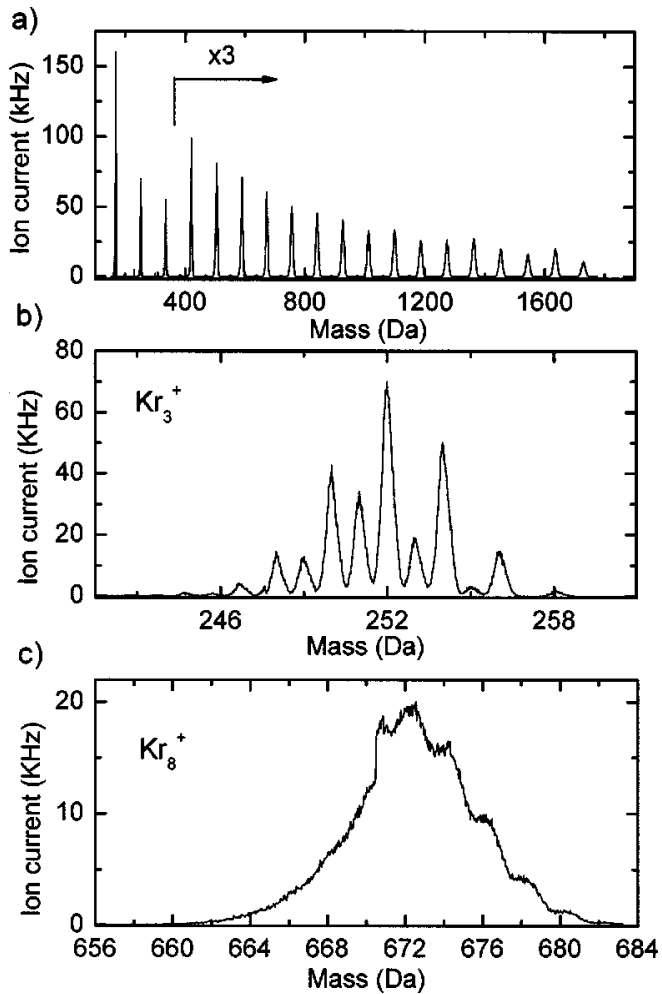

FIG. 2. Mass spectrum of krypton clusters: (a) $\mathrm{Kr}_{n}^{+} n \leqslant 20$; (b) $\mathrm{Kr}_{3}^{+}$; (c) $\mathrm{Kr}_{8}^{+}$.

(last two rows in Table I), the fragment ion peaks correspond to the loss of ${ }^{84} \mathrm{Kr},{ }^{83} \mathrm{Kr},{ }^{86} \mathrm{Kr},{ }^{82} \mathrm{Kr},{ }^{80} \mathrm{Kr}$, and ${ }^{78} \mathrm{Kr}$, in that order.

The solid line represents a nonlinear least squares fit of three sets of six Gaussians each (one for the loss of each isotope), with a total number of four free parameters: three amplitudes and the fourth being the width of the peaks; all other parameters (peak positions and relative amplitudes

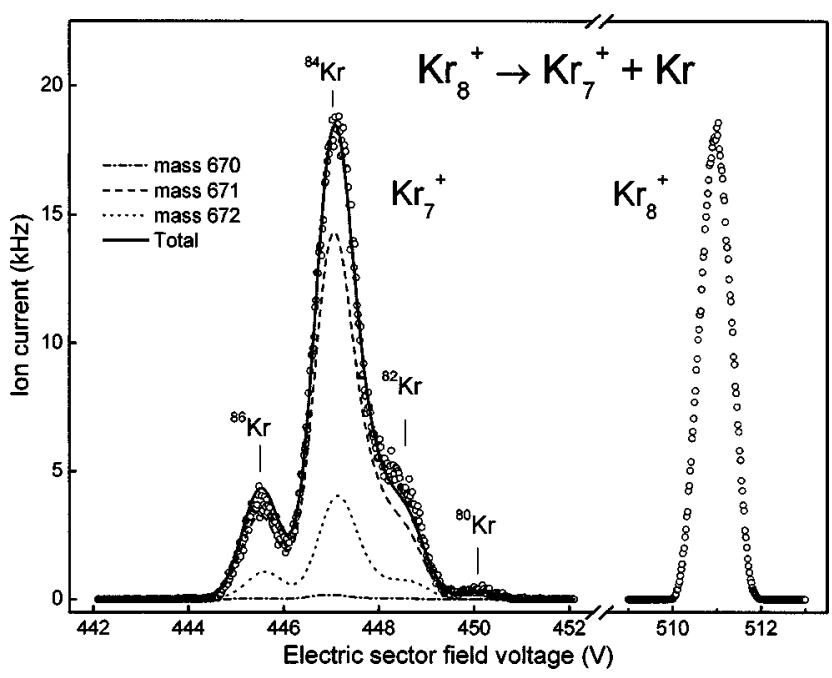

FIG. 3. MIKE scan for metastable decay of $\mathrm{Kr}_{8}^{+}$(mass $671 \mathrm{Da}$ ) into $\mathrm{Kr}_{7}^{+}$ $+\mathrm{Kr}$. The multiplet structure in the fragment ion peak arises from loss of six different $\mathrm{Kr}$ isotopes; some of them are indicated by lines. The solid line indicates least-square fits of Gaussians with the relative probabilities of the six isotopes being computed from the isotopic abundance. See text for details. within each set) were computed as discussed in the preceding section. The dashed line shows the main contribution arising from decay of $\mathrm{Kr}_{8}^{+}$at $671 \mathrm{Da}$, while the dashed-dotted and dotted lines show the contributions from neighboring parent ions at 670 and $672 \mathrm{Da}$, respectively. From the width $w$ $\pm \Delta w$ we derive the $\langle\mathrm{KER}\rangle \pm \Delta\langle\mathrm{KER}\rangle$, and from this quantity we derive the transition state temperature, isokinetic heat bath temperature, and the binding energy with the corresponding error bars.

Our experimental results indicate that unimolecular decay of very small rare gas cluster ions, of size $n=10$ or smaller, is not properly described by the formalism in the preceding section, partly because their decay is not purely due to vibrational predissociation (see also the nonstatistical decay of dimer ions ${ }^{31}$ ), and partly because the evaporative ensemble model $^{10}$ fails for small sizes. For example, the shapes of metastable peaks (which are fully resolved for small cluster size) are not strictly Gaussian, and the $\langle\mathrm{KER}\rangle$ exhibits a dependence on stagnation temperature (see below). Similar observations have been made by Magnera et $a l .{ }^{16}$ when they measured metastable fractions for the decay of nitrogen and mixed argon/nitrogen cluster ions. Also in our laboratory we found recently when we analyzed oxygen cluster ions ${ }^{17}$ that both, average kinetic energy release $\langle\mathrm{KER}\rangle$ data derived from the peak shapes and the time dependence of the metastable fractions show a characteristic dependence on cluster size indicating a change in the metastable fragmentation mechanism when going from the dimer to the decamer ion. Because of all these facts the analysis outlined above for determining binding energies is only applied to cluster ions of size $n \geqslant 10$.

In Fig. 4 we present the size dependence of the $\langle\mathrm{KER}\rangle$ for neon, argon, and krypton cluster ions including error bars, which have been obtained by taking the error of the fit parameter $w$, i.e., the width of the peak, and then calculating the standard deviation for the corresponding $\langle\mathrm{KER}\rangle$ value. From these values we have derived binding energies for the cluster ions $\operatorname{Rg}_{n}^{+}$with $n \geqslant 10$. It can be clearly seen that the present neon and argon data (designated as stars and full circles) smoothly extend the earlier measurements ${ }^{14,15}$ (designated as open circles) carried out with the two sector field apparatus (the differences between the present and earlier $\mathrm{Kr}$ data are due to the deficiencies outlined for the two sector field mass spectrometer used in the earlier analysis). However, for small neon cluster ions one can observe a significant discrepancy in the $\langle\mathrm{KER}\rangle$ between the results obtained with the two experimental setups.

In order to understand this difference we investigated in detail the influence of the cluster production conditions. These additional experiments were performed with argon, because this element is essentially monoisotopic, and less expensive than neon and krypton. It turned out that the stagnation temperature has an influence on the $\langle\mathrm{KER}\rangle$ of small cluster ions, i.e., for higher stagnation temperatures the cluster ions decay with a significantly smaller $\langle$ KER $\rangle$ compared to lower stagnation temperatures. In Fig. 5 one can see that this effect changes the $\langle\mathrm{KER}\rangle$ by about $40 \%$ for the reaction $\mathrm{Ar}_{6}^{+} \rightarrow \mathrm{Ar}_{5}+\mathrm{Ar}$ (upper panel), but only by $6 \%$ for $\mathrm{Ar}_{10}^{+}$ $\rightarrow \mathrm{Ar}_{9}+\mathrm{Ar}$ (lower panel). In our earlier studies with the two 


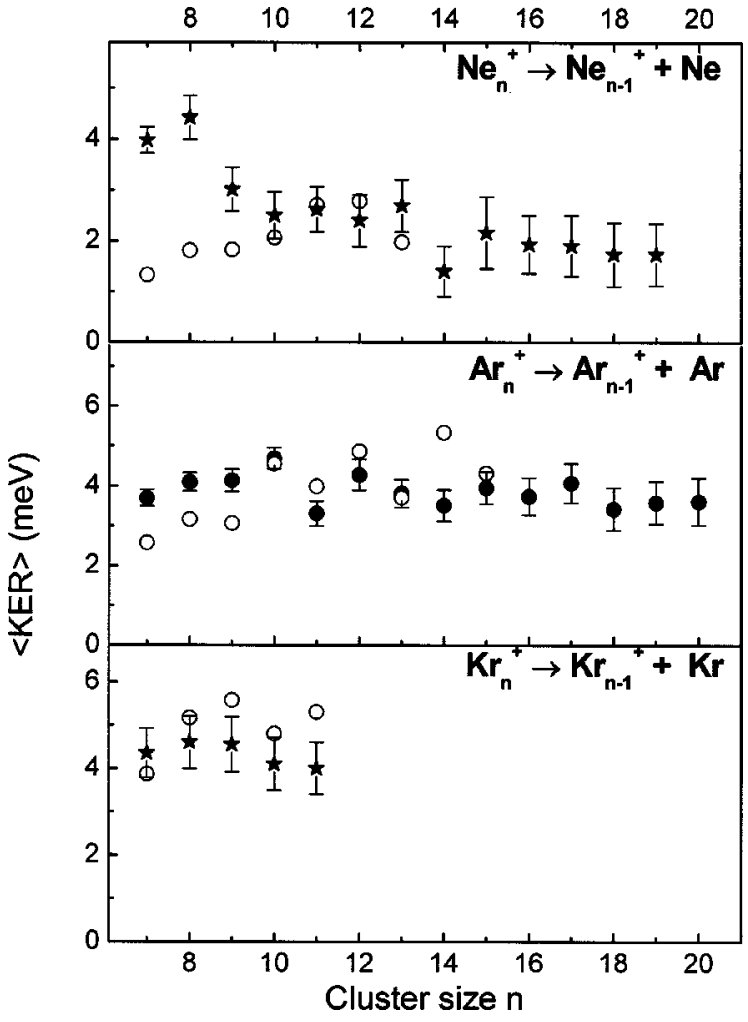

FIG. 4. Average kinetic energy release $\langle$ KER $\rangle$ for metastable monomer evaporation of neon, argon, and krypton cluster ions. Full stars and full circles represent measurements using the three sector field mass spectrometer, open circles represent earlier measurements with a two sector field mass spectrometer (Refs. 14, 15), see text.

sector field machine we had used relatively high stagnation temperatures compared to the present studies. With the three sector field machine used in the present experiment we need to work at smaller temperatures in order to produce more parent ions because (i) the decay rate is smaller in ff3 (later time window) and (ii) we lose intensity at the narrow slits, which provide the high mass and energy resolution. Therefore one can conclude that the difference in the $\langle\mathrm{KER}\rangle$ values

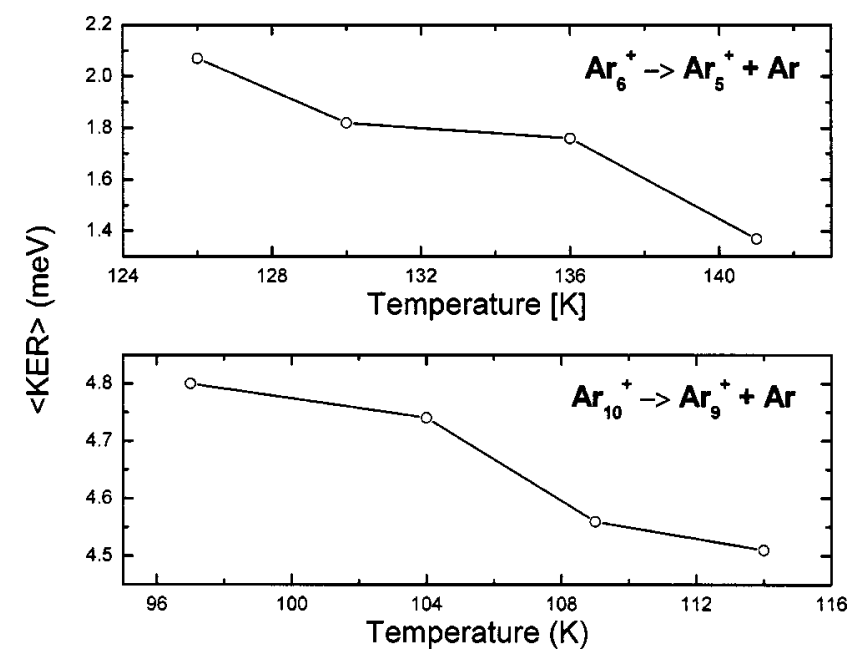

FIG. 5. 〈KER $\rangle$ plotted as a function of the stagnation temperature of the cluster source showing the influence of different production conditions in the case of $\mathrm{Ar}_{6}^{+}$(upper panel) and $\mathrm{Ar}_{10}^{+}$(lower panel).

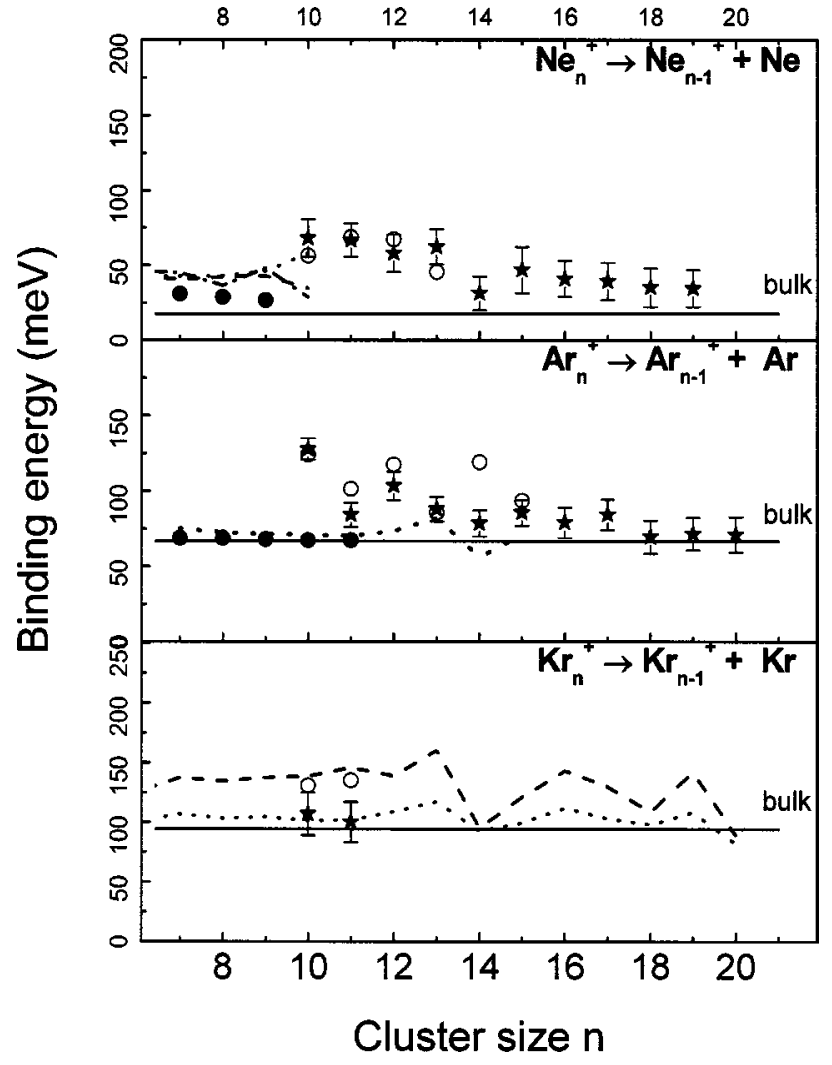

FIG. 6. Full stars: Binding energies of $\mathrm{Ne}, \mathrm{Ar}$, and $\mathrm{Kr}$ cluster ions calculated from the $\langle\mathrm{KER}\rangle$ measured with the three sector field mass spectrometer. Open circles: BE calculated from measurements using the two-sector field mass spectrometer. Full dots: Results from high-pressure gas phase experiments (Refs. 19, 21). Solid horizontal lines: Enthalpy of vaporization of the bulk (Ref. 32). Dashed, dotted, and dot-dashed lines in top panel: Binding energies calculated by (Ref. 20) for $\mathrm{Ne}_{n}^{+}$using a DIM model, a DIM model including dimer-atom interaction, and ab initio methods, respectively. Dotted line middle panel: Binding energies for $\mathrm{Ar}_{n}^{+}$using Monte Carlo calculations (Ref. 22). Dashed and dotted lines in lowest panel: Calculated binding energies (Ref. 23) for $\mathrm{Kr}_{n}^{+}$using the DIM+SO, and DIM+SO+ID-ID, respectively.

measured with the two setups is probably caused by different production conditions. As already mentioned above we did not carry out the same analysis with neon and krypton cluster ions, but we would expect similar findings.

Binding energies for neon, argon and krypton cluster ions are shown in Fig. 6 together with theoretical and other experimental data. In the top panel of Fig. 6 we show the experimental results for the binding energies of $\mathrm{Ne}_{10}^{+}$to $\mathrm{Ne}_{19}^{+}$. Also shown are values computed by Gianturco and co-workers ${ }^{20}$ for $\mathrm{Ne}_{n}^{+}$with the $\mathrm{D}$ (iatomic)I(n)M(olecule) method (dashed line), the DIM method including dimer atom potentials (dotted line), and ab initio (dot-dashed line). Unfortunately we cannot compare the experimental with the theoretical values, because they are for different cluster sizes. However, it is apparent that the binding energies determined in this work approach the bulk value ${ }^{32}$ as expected. In addition, Hiraoka et al. ${ }^{19}$ have obtained in a high pressure gas phase experiment binding energies for clusters of size $n \leqslant 9$ (designated as full circles), which seem to give much smaller BEs as ours if we extrapolate our results to smaller sizes.

In the case of argon we can compare our results with 
Monte Carlo calculations by Böhmer and Peyerimhoff ${ }^{22}$ (dotted line) which assumed a trimeric ion core within the argon cluster ions, and a vibrational temperature of $10 \mathrm{~K}$. Our experimental results are higher for all cluster sizes, but again nicely approach the corresponding bulk value. ${ }^{32}$ The comparison with experimental results by Hiraoka et al. ${ }^{21}$ (designated as full circles) is difficult because only $\mathrm{Ar}_{10}^{+}$and $\mathrm{Ar}_{11}^{+}$have been investigated by both methods

For krypton clusters we compare the present results with binding energies calculated by Kalus et al. $;^{23}$ no other experimental values are available for the cluster sizes studied by us. Kalus et al. used two slightly different approaches to calculate binding energies. The dashed curve in Fig. 6 (lowest panel) are results based on a diatomic in molecule interaction including the spin-orbit interaction $(\mathrm{DIM}+\mathrm{SO})$, whereas the dotted line also includes the polarization three body forces $(\mathrm{DIM}+\mathrm{SO}+\mathrm{ID}-\mathrm{ID})$. It is difficult to say which model agrees better with our data, because our results obtained with the two sector field machine agree better with the $\mathrm{DIM}+\mathrm{SO}$ calculation, whereas the results obtained with the improved experimental setup favors the DIM+SO+ID-ID model. Due to the better experimental conditions used in the present work we conclude that the DIM+SO+ID-ID describes the krypton cluster ions better. Anyhow, the binding energies of these cluster sizes are close to the enthalpy of vaporization of the bulk. ${ }^{32}$

In conclusion, the application of three sector field mass spectrometry and an advanced method of data analysis, where the contributions from all isotopomers is quantitatively taken into account, enable us to extend earlier data to higher sizes. For all three rare gases studied, we could measure and analyze highly resolved kinetic energy release distributions and from those results derive binding energies, which are found to approach the enthalpy of vaporization of the condensed phase.

\section{ACKNOWLEDGMENTS}

We would like to thank Professor I. Paidarova for providing us with their unpublished results on the modeling of structure and energetics of krypton clusters ions. Work has been partially supported by the FWF, ÖAW, and ÖNB, Wien, Austria, and the European Commission, Brussels.
${ }^{1}$ T. D. Märk and O. Echt, Cluster of Atoms and Molecules II, edited by H. Haberland (Springer-Verlag, Berlin, 1994), Vol. 56, p. 154.

${ }^{2}$ O. K. Rice and H. C. Ramsperger, J. Am. Chem. Soc. 49, 1617 (1927).

${ }^{3}$ L. S. Kassel, J. Phys. Chem. 32, 225 (1928).

${ }^{4}$ J. Laskin and C. Lifshitz, Chem. Phys. Lett. 277, 564 (1997).

${ }^{5}$ S. Tomita, J. U. Andersen, C. Gottrup, P. Hvelplund, and U. V. Pedersen, Phys. Rev. Lett. 87, 073401 (2001).

${ }^{6}$ P. C. Engelking, J. Chem. Phys. 87, 936 (1987).

${ }^{7}$ A. J. Stace, J. Chem. Phys. 85, 5774 (1986).

${ }^{8}$ A. J. Stace and A. K. Shukla, Chem. Phys. Lett. 85, 157 (1982).

${ }^{9}$ S. Wei, W. B. Tzeng, and A. W. Castleman, J. Chem. Phys. 92, 332 (1990).

${ }^{10}$ C. E. Klots, J. Phys. Chem. 92, 5864 (1988).

${ }^{11}$ S. Wei, K. Kilgore, W. B. Tzeng, and A. W. Castleman, J. Phys. Chem. 95, 8306 (1991)

${ }^{12}$ S. Wei, Z. Shi, and A. W. Castleman, J. Chem. Phys. 94, 8604 (1991).

${ }^{13}$ C. Lifshitz and F. Louage, Int. J. Mass Spectrom. Ion Processes 101, 101 (1990).

${ }^{14}$ R. Parajuli, S. Matt, O. Echt, A. Stamatovic, P. Scheier, and T. D. Märk, Eur. Phys. J. D 16, 69 (2001).

${ }^{15}$ R. Parajuli, S. Matt, O. Echt, A. Stamatovic, P. Scheier, and T. D. Märk, Chem. Phys. Lett. 352, 288 (2002).

${ }^{16}$ T. F. Magnera, D. E. David, and J. Michl, J. Chem. Soc., Faraday Trans. 86, 2427 (1990).

${ }^{17}$ S. Matt, R. Parajuli, A. Stamatovic, P. Scheier, and T. D. Märk, J. Chem. Phys. 116, 7583 (2002).

${ }^{18}$ S. Matt-Leubner, A. Stamatovic, R. Parajuli, P. Scheier, T. D. Märk, O. Echt, and C. Lifshitz, Int. J. Mass. Spectrom. 222, 213 (2003).

${ }^{19}$ K. Hiraoka and T. Mori, J. Chem. Phys. 92, 4408 (1990).

${ }^{20}$ F. Sebastianelli, E. Yurtsever, and F. A. Gianturco, Int. J. Mass. Spectrom. 220, 193 (2002).

${ }^{21}$ K. Hiraoka and T. Mori, J. Chem. Phys. 90, 7143 (1989).

${ }^{22}$ H. U. Böhmer and S. D. Peyerimhoff, Z. Phys. D: At., Mol. Clusters 8, 239 (1989).

${ }^{23}$ R. Kalus, I. Paidarova, D. Herivnak, P. Paska, and F. X. Gadea, Chem. Phys. 294, 141 (2003).

${ }^{24}$ R. G. Cooks, J. H. Beynon, R. M. Caprioli, and G. R. Lester, Metastable Ions (Elsevier, Amsterdam, 1973).

${ }^{25}$ J. Holmes and A. D. Osborne, Int. J. Mass Spectrom. Ion Phys. 23, 189 (1977).

${ }^{26}$ M. Guilhaus, R. K. Boyd, A. G. Brenton, and J. H. Beynon, Int. J. Mass Spectrom. Ion Processes 67, 209 (1985).

${ }^{27}$ W. Forst, Theory of Unimolecular Reactions (Academic, New York, 1973).

${ }^{28}$ C. E. Klots, Z. Phys. D: At., Mol. Clusters 21, 335 (1991).

${ }^{29}$ C. E. Klots, Z. Phys. D: At., Mol. Clusters 20, 105 (1991).

${ }^{30}$ K. Głuch, S. Matt-Leubner, O. Echt, R. Deng, J. U. Andersen, P. Scheier, and T. D. Märk, Chem. Phys. Lett. (in press).

${ }^{31}$ J. Fedor, R. Parajuli, S. Matt-Leubner, O. Echt, F. Hagelberger, K. Gluch, M. Probst, P. Scheier, and T. D. Märk, Phys. Rev. Lett. 91, 133401 (2003).

${ }^{32}$ D. R. Lide, CRC Handbook of Chemistry and Physics (CRC, Boca Raton, 2000). 